\title{
SISTEMATIZAÇÃO DA ASSISTÊNCIA DE ENFERMAGEM: UMA DÉCADA DE IMPLEMENTAÇÃO SOB A ÓTICA DO ENFERMEIRO
}

\section{SYSTEMATIZATION OF NURSING CARE: A DECADE OF IMPLEMENTATION UNDER THE NURSE'S VIEWPOINT}

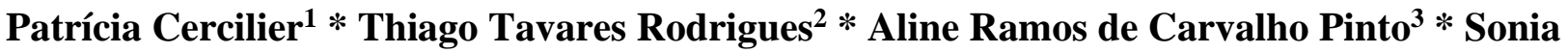 \\ Regina de Souza ${ }^{4}$
}

\begin{abstract}
RESUMO
Introdução: A Sistematização da Assistência de Enfermagem foi instituída no Brasil pela resolução 358-2009, para organizar o trabalho profissional e operacionalizar o processo de Enfermagem. Desta forma a SAE é essencial para uma assistência qualificada e para o reconhecimento da profissão enquanto ciência. Objetivos: Identificar e apresentar o perfil das produções científicas dos Enfermeiros na última década (2009- 2019) sobre a SAE; e apontar avanços e os limites da SAE. Método: trata-se de uma revisão integrativa, foram utilizados como descritores: Processo de Enfermagem; Diagnóstico de Enfermagem; Enfermagem. Como critério de exclusão: artigos que não tiveram relação com o tema, artigos duplicados em outras bases de dados, teses, dissertações e estudos de revisão. o recorte temporal foi de (2009- 2019). Resultados: Após leitura dos artigos foram identificados dois eixos temáticos: 1. Avanços e benefícios da aplicação da SAE, neste destacou-se os ganhos gerados para a profissão a partir da sua aplicabilidade, como a autonomia e a qualificação da assistência; 2. Limites e fragilidades na aplicação da SAE, foram identificados como limitações: o dimensionamento de enfermagem inadequado, e a carência de atualizações e educação permanente. Conclusão: A SAE é uma grande conquista para a Enfermagem, a partir da sua implementação inúmeros avanços foram alcançados. No entanto há barreiras a serem ultrapassadas, que dificultam a implementação como: dimensionamento de pessoal, treinamento e capacitação, interferindo diretamente na qualidade dos registros e da assistência. Ressaltamos a importância de um olhar atentivo das instituições, dos órgãos de classe e de todos os envolvidos.
\end{abstract}

Palavras-chave: Processo de Enfermagem; Diagnóstico de Enfermagem; Enfermagem.

\begin{abstract}
Introduction: Nursing Assistance Systematization was instituted in Brazil by resolution 358-2009, to organize professional work and operationalize the Nursing process. In this way, SAE is essential for qualified assistance and for the recognition of the profession as a science. Objectives: To identify and present the profile of the scientific production of Nurses in the last decade (2009- 2019) on SAE; and point out the advances and limits of SAE. Method: this is an integrative review; the following descriptors were used: Nursing Process; Nursing Diagnosis; Nursing. As exclusion criteria: articles that were not related to the theme, duplicate articles in other databases, theses, dissertations and review studies. the time frame was (2009-2019). Results: After reading the articles, two thematic axes were identified: 1. Advances and benefits of the application of SAE, in this stand out the gains generated for the profession from its applicability, such as autonomy and qualification of assistance; 2. Limits and weaknesses in the application of SAE, were identified as limitations: the inadequate dimensioning of nursing, and the lack of updates and permanent education. Conclusion: SAE is a great achievement for Nursing, since its implementation numerous advances have been achieved. However, there are barriers to be overcome, which hinder implementation, such as: staff sizing, training and qualification, directly interfering with the quality of records and assistance. We emphasize the importance of an attentive look at institutions, professional bodies and all those involved.
\end{abstract}

Keywords: Nursing Process; Nursing Diagnosis; Nursing.

\footnotetext{
${ }^{1}$ Mestranda em Enfermagem pela Universidade do Estado do Rio de Janeiro. Residência em Clínica médica e Cirúrgica pela Universidade Federal do Estado do Rio de Janeiro. Enfermeira Especialista em Enfermagem em Terapia Intensiva pela Universidade Federal do Rio de Janeiro. ORCID: https://orcid.org/0000-0002-4277-304X/print

2 Residência em Enfermagem Clínica médica e Cirúrgica pela Universidade Federal do Estado do Rio de Janeiro(UNIRIO).Bacharelado em Enfermagem pela Universidade (UNIGRANRIO). Email: enfthiagotavares@yahoo.com.br ORCID: https://orcid.org/0000-0001-8884-9882/print

${ }_{3}$ Mestre em Enfermagem pela Universidade Federal do Estado do Rio de Janeiro(UNIRIO). Enfermeira líder do Hospital Universitário Clementino Fraga Filho - (UFRJ). ORCID: https://orcid.org/0000-0002-9610-1898/print

${ }^{4}$ Doutora em Enfermagem. Professora associada do Depto de Enfermagem Médico Cirúrgica da Escola de Enfermagem Alfredo Pinto (UNIRIO). ORCID: http://orcid.org/0000-0001-7981-0038
} 


\section{INTRODUÇÃO}

O Processo de enfermagem (PE) é idealizado desde Florence Nightingale que participou como voluntária na Guerra da Criméia em 1854, que preconizava que as enfermeiras deveriam estar submetidas a uma forte organização disciplinar, além de enfatizar que as enfermeiras deveriam ser ensinadas a fazer observações e julgamentos. Embora a terminologia PE não existir naquela época, a recomendação de Florence idealiza o conceito que temos a respeito deste instrumento de trabalho ${ }^{(1)}$.

O emprego do processo de enfermagemno Brasil foi promovido pelo estudo de Wanda Aguiar Horta, na década de 70, com base no referencial das Teorias das Necessidades Humanas Básicas deMaslow, noqual a assistência de Enfermagem deveria apoiar-se em uma metodologia científica. Que definiu o PE como a dinâmica das ações sistematizadas e inter-relacionadas, visandoà assistência ao ser humano e caracteriza-se pelo inter-relacionamento e dinamismo de suas fases ou passos ${ }^{(2-3)}$.

Em 2009 O Conselho Federal de Enfermagem publicou a resolução 358/2009 que dispõe sobre Sistematização da Assistência de Enfermagem (SAE), e implementa o Processo de Enfermagem. Resolvendo quePE deve ser realizado, de modo deliberado e sistemático, em todos os ambientes, públicos ou privados, em que ocorre o cuidado profissional de Enfermagem. Além de trazer a diferenciação de SAE e PE ${ }^{(2-}$ 3).

De acordo com esta resolução a SAE organiza o trabalho profissional quanto ao método, pessoal, e instrumentos, tornando possível a operacionalização do processo de Enfermagem, enquanto o PE é um instrumento metodológico que orienta o cuidado profissional de Enfermagem e a documentação da prática profissional. Considerando que a operacionalização e documentação do PE evidencia a contribuição da Enfermagem na atenção à saúde da população, aumentando a visibilidade e o reconhecimento do profissional. O Processo de Enfermagem organiza-se em cinco etapas inter-relacionadas, interdependentes e recorrentes: coleta de dados (ou Histórico de Enfermagem; Diagnóstico de Enfermagem; Planejamento de Enfermagem; Implementação; Avaliação de enfermagem) $)^{(4)}$. Compreendemos que para o cumprimentoda implementação da SAE, a partir da Resolução supracitada, que data de uma década, foram necessária capacitação profissional e adequação técnico-científicas nas instituições de saúde públicas e privadas além do fortalecimento na reprodução da SAE 
nas grades curriculares das instituições de ensino.

Deste modo, acreditamos que a forma que as instituições se adequaram para dar visibilidade a SAE, o processo de capacitação do Enfermeiro, e a importância atribuída pelo Corpo de Enfermagem são fatores a serem considerados. Sendo assim, acreditamos que os fatores relevantes à aplicabilidade da SAE, seja alvo de inquietações do Enfermeiro.

Este estudo tem como objetivos identificar as produções científicas do Enfermeiro na última década sobre a SAE; apresentar o perfil da produção do Enfermeiro na última década sobre a SAE e apontar os avanços e os limites da sistematização da assistência de enfermagem.

$\mathrm{O}$ estudo justifica-se pelo significado que a Sistematização da Assistência de Enfermagem representa para os profissionais quanto à validação da profissão e pelo reconhecimento do papel do enfermeiro nas diversas áreas de atuação. Uma vez após 10 anos de sua implementação, precisamos compreender qual foi seu papel durante esta trajetória para fortalecimento da profissão e refletir sobre as perspectivas futura.

Consideramos que este estudo é relevante, pois irá contribuir com a comunidade cientifica e acadêmica, por apresentar o panorama da SAE nos diferentes cenários de Saúde e subsidiar discussões quanto à eficiência da sua aplicabilidade e/ou fragilidades, sob a ótica do Enfermeiro.

\section{METODOLOGIA}

Há inúmeros caminhos para refletir sobre a produção de um conhecimento de uma área. Este estudo trata-se de uma revisão integrativa. De acordo com Mendes, Silveira e Galvão (2008) este método de pesquisa realiza junções de diversos estudos que abordem a um determinado assunto ou prática, através de uma metodologia organizada. Com isso, possibilita a síntese sobre tal conhecimento que auxilia aos profissionais de saúde no direcionamento de uma prática eficaz, pautada na Prática Baseada Evidências ${ }^{(5)}$.

$$
\text { Para a construção da revisão }
$$
integrativa é preciso percorrer seis etapas. 1) identificação do tema e seleção da hipótese ou questão de pesquisa para a elaboração da revisão integrativa; 2) estabelecimento de critérios para inclusão e exclusão de estudos/ amostragem ou busca na literatura; 3) definição das informações a serem extraídas dos estudos selecionados/ categorização dos estudos;4) avaliação dos estudos incluídos na revisão integrativa; 5) interpretação dos resultados; 6) apresentação da revisão/síntese do conhecimento ${ }^{(5)}$. 
Para a realização da primeira etapa, estabeleceu-se como questão norteadora do estudo:O que os enfermeiros produziram nos últimos dez anos sobre a sistematização da assistência de enfermagem?

$\mathrm{Na}$ segunda etapa foram estabelecidos os critérios de inclusão:artigos publicados no recorte temporal de 2009 a 2020, no idioma português, com texto completo disponível, nas bases de dados LILACS (Literatura Latino-Americana e do Caribe em Ciências da Saúde), BDENF (Base de dados de Enfermagem) e MEDLINE. Os critérios de exclusão adotados foram: artigos que não tiverem relação com o tema, artigos duplicados em outras bases dados, teses, dissertações e estudos de revisão.

$\mathrm{Na}$ terceira etapa a busca foi realizada nada Biblioteca Virtual de Saúde (BVS), utilizando os bancos de dados LILACS (Literatura Latino-Americana e do Caribe em Ciências da Saúde), BDENF (Base de dados de Enfermagem) e MEDLINE. Os descritores adotados foram consultados nos Descritores de Ciências de Saúde (DECS): "Processo de Enfermagem", "Diagnóstico de Enfermagem" associados utilizando o operador booleano and. Conforme mostra a Figura 1.

Figura 1: Fluxograma da busca dos artigos na BVS. Rio de Janeiro, RJ, Brasil, 2020.

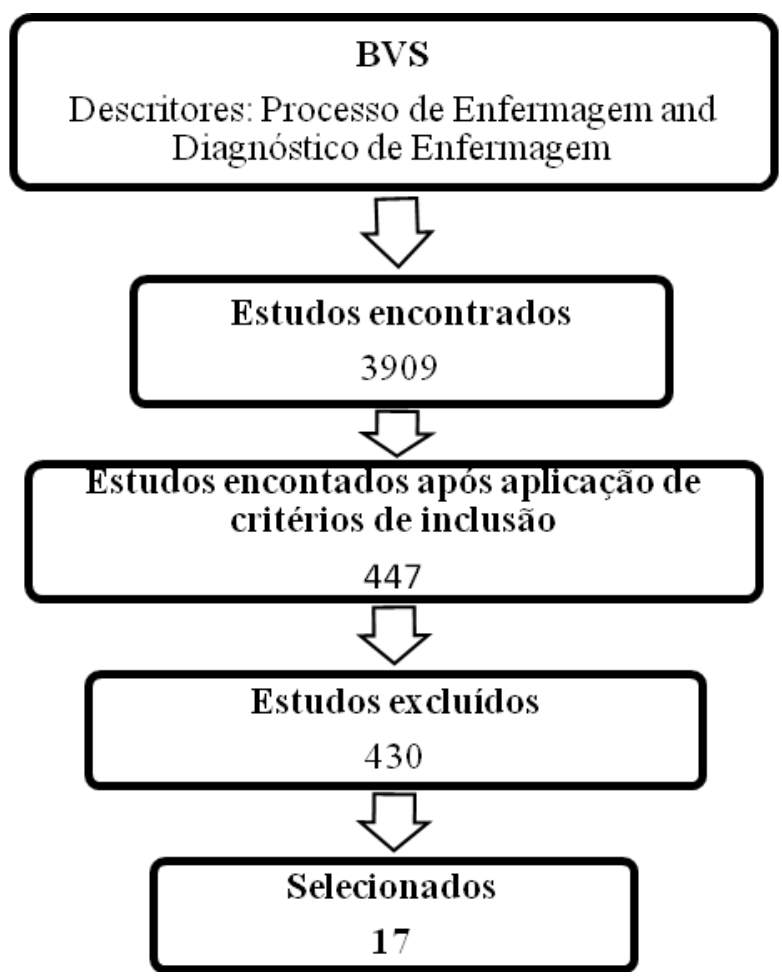

Fonte:Elaborado pelos próprios autores. 
A análise qualitativa dos conteúdos dos artigos foi a partir da técnica de análise temática, descrita d e acordo com BARDIN, o termo análise temática é basicamente a contagem, evidenciação de um ou vários temas ou itens de significação, contido em uma unidade de codificação previamente selecionada ${ }^{(6)}$.

De acordo com Bardin, o tema é uma unidade de significação que se liberta do texto analisado e pode ser traduzido por um resumo, por uma frase ou por uma palavra. Ainda para a autora, para chegar-se ao tema, faz-se necessário "descobrir os 'núcleos de sentido' que compõem a comunicação e cuja presença, ou frequência de aparição, pode significar alguma coisa para o objetivo analítico escolhido" (Bardin, 2011, p. 105). Com essa técnica, pode-se caminhar, também, na direção da "descoberta do que está por trás dos conteúdos manifestos, indo além das aparências do que está sendo analisado" (Bardin, 2011, p. 74).

$\mathrm{Na}$ análise temática de Bardin, o núcleo de sentido e tema integram um mesmo processo analítico, sendo o primeiro o ponto de partida para estabelecer-se o segundo ${ }^{(6)}$. Nesta revisão integrativa, o tema está sendo entendido como uma categoria mais ampla que pode abranger mais de um núcleo de sentido.

$\mathrm{Na}$ análise dos artigos, buscou-se a identificação das ideias centrais dos artigos, passando pela interpretação dos sentidos dessas ideias e pelo agrupamento das ideias em categorias empíricas ou núcleos de sentido, chegando-se a descrição de temas, como classificações mais amplas.

Em síntese, foram empregados os seguintes passos de análise: (a) leitura exaustiva de cada artigo visando a uma compreensão global e à descoberta da abordagem utilizada pelos seus autores; (b) identificação das ideias centrais de cada artigo; (b) classificação das ideias em torno de núcleos de sentido; (c) comparação entre os diferentes núcleos de sentido presentes nos artigos estudados; (d) classificação dos núcleos de sentido em eixos mais abrangentes (temas) em torno dos quais situam-se as discussões dos autores e (e) redação das sínteses interpretativas de cada tema.

\section{RESULTADOS}

Após a busca na BVS com os critérios aplicados, foram selecionados 17 artigos, que corresponderam aos objetivos propostos para a síntese desta revisão integrativa. Para o mapeamento dos artigos encontrados, foi elaborado um quadro para facilitar o processo de identificação dos artigos, com codificação dos artigos em número, título, autores, local, ano e revista de publicação. Conforme mostra quadro 1. 
Quadro 1- Publicações por codificação artigo em número, título, autores, local, ano, revista de publicação. Rio de Janeiro, RJ, Brasil, 2020.

\begin{tabular}{|c|c|c|c|c|}
\hline Artigo & Título & Autores & Local-Ano & Revista \\
\hline 1 & $\begin{array}{l}\text { Análise da implementação da } \\
\text { Sistematização da Assistência de } \\
\text { Enfermagem em uma unidade de } \\
\text { reabilitação }\end{array}$ & Neves R S & $\begin{array}{l}\text { Distrito Federal, } \\
2010\end{array}$ & REBEn \\
\hline 2 & $\begin{array}{l}\text { Tempo despendido na execução do } \\
\text { processo de enfermagem em um } \\
\text { centro de tratamento intensivo }\end{array}$ & $\begin{array}{l}\text { Almeida M A } \\
\text { et. al }\end{array}$ & $\begin{array}{l}\text { Rio Grande do } \\
\text { Sul, } 2012\end{array}$ & $\begin{array}{l}\text { Esc. Anna } \\
\text { Nery }\end{array}$ \\
\hline 3 & $\begin{array}{l}\text { Sistematização da Assistência de } \\
\text { Enfermagem para acompanhamento } \\
\text { ambulatorial de pacientes com } \\
\text { esclerose múltipla }\end{array}$ & $\begin{array}{l}\text { Corso, N A } \\
\text { Aet al }\end{array}$ & Fortaleza, 2013 & $\begin{array}{l}\text { RevEscenfe } \\
\text { rm USP }\end{array}$ \\
\hline 4 & $\begin{array}{l}\text { Construção de um software com o } \\
\text { Processo de Enfermagem em terapia } \\
\text { intensiva }\end{array}$ & $\begin{array}{l}\text { Martins M C } \\
\text { T M et al, } \\
\text { Chianca T C } \\
\text { M }\end{array}$ & $\begin{array}{l}\text { Minas Gerais, } \\
2016\end{array}$ & $\begin{array}{l}\text { J. Health } \\
\text { Inform }\end{array}$ \\
\hline 5 & $\begin{array}{l}\text { Processo de enfermagem aplicado a } \\
\text { paciente submetida à histerectomia: } \\
\text { relato de experiência }\end{array}$ & $\begin{array}{l}\text { Rocha R C et } \\
\text { al }\end{array}$ & $\begin{array}{l}\text { Piauí } \\
2015\end{array}$ & $\begin{array}{l}\text { RevEnf } \\
\text { UFPI }\end{array}$ \\
\hline 6 & $\begin{array}{l}\text { Sistematização da assistência de } \\
\text { enfermagem à criança e ao adolescente } \\
\text { em sofrimento psíquico }\end{array}$ & $\begin{array}{l}\text { Monteiro A } \\
\text { R M et al }\end{array}$ & Fortaleza 2015 & $\begin{array}{l}\text { J. res: } \\
\text { fundam care }\end{array}$ \\
\hline 7 & $\begin{array}{l}\text { Aplicação do Processo de } \\
\text { Enfermagem: Estudo de caso com um } \\
\text { portador do vírus da hepatite C }\end{array}$ & $\begin{array}{l}\text { Silva R S et } \\
\text { al }\end{array}$ & $\begin{array}{l}\text { Salvador } \\
2010\end{array}$ & $\begin{array}{l}\text { Rev Baiana } \\
\text { de Enf }\end{array}$ \\
\hline 8 & $\begin{array}{l}\text { Processo de Enfermagem ao } \\
\text { adolescente portador de anemia } \\
\text { falciforme }\end{array}$ & $\begin{array}{l}\text { Gomes I P et } \\
\text { al }\end{array}$ & $\begin{array}{l}\text { Paraíba } \\
2011\end{array}$ & $\begin{array}{l}\text { RevBrasCie } \\
\text { n Saúde }\end{array}$ \\
\hline 9 & A aplicação do processo de & Schmalfuss J & Rio Grande do & RevEnfUFP \\
\hline
\end{tabular}


enfermagem no atendimento a uma

M; Scholz D Sul

$\mathrm{E}$

mulher com artrite reumatoide

C S; Prates L 2013

A

10 Sistematização da assistência de

Toledo V P; São Paulo

Rev Baiana

enfermagem em unidade de internação

Motobu S N; 2015

de Enf

psiquiátrica

Garcia A P R

F

11 Processo de enfermagem aplicado a Mattos C M Porto Alegre Estud. idosos com Alzheimer que participam Z et al $2011 \quad$ interdiscipl. do projeto estratégias de reabilitação envelhec

12 Sistematização da Assistência de Truppel T C Paraná

REBEn

Enfermagem em unidade de terapia

et al

2009 intensiva

13 Sistematização da Assistência de

$\begin{array}{lll}\text { Kamimura H } & \text { São Paulo } & \text { REBEn } \\ \text { K; Paiva B S } & 2009 & \\ \text { B; Ayres J A } & & \end{array}$

Enfermagem: acidente por

14 Desafios encontrados na realização da Felix N N; São Paulo ArqCiênc sistematização da assistência de

Rodrigues C 2009

Saúde enfermagem (SAE) em unidade de

D S; Oliveira

pronto atendimento

V D C

15 Sistematização da assistência de Junior E F P Rio de Janeiro Revenferm enfermagem aplicada a um et al 2015

UERJ adolescente hospitalizado por paracoccidioidomicose

16 Instrumento de Sistematização da

$\begin{array}{lll}\text { Marques D K } & \text { Paraíba } & \text { Rev. Min. } \\ \text { A; Nóbrega } & 2009 & \text { Enferm } \\ \text { MML } & & \end{array}$
adolescentes hospitalizados

Toledo V P; São Paulo

REBEn

17 Processo de Enfermagem para

Ramos N A; 2010

pacientes com Anorexia Nervosa

WopereisF

Fonte: Elaborado pelos próprios autores. 
Para realização da análise dos artigos facilitar a síntese dos dados coletados. selecionados foi elaborado o instrumento para

Quadro 2 - Instrumento para a coleta de dados com código do artigo, avanços e limites. Rio de Janeiro, RJ, Brasil, 2020.

\section{Artigo}

\section{Avanços / Limites}

1 Apesar da existência de formulários que facilitam a implementação da SAE e a evolução de enfermagem padronizada, os enfermeiros possuem dificuldades para operacionalização da SAE. Evidenciou-se a fragmentação do registro e da aplicação da SAE, devido à necessidade de recursos humanos e educação permanente.

2 Identificou-se a necessidade do planejamento de recursos humanos visando à assistência qualificada, considerando que, a aplicação do PE demanda um tempo variável para a sua execução e há a necessidade de um quantitativo adequado de enfermeiros.

3 A aplicação efetiva do processo de enfermagem aos pacientes ambulatoriais com esclerose múltipla permite diagnosticar as necessidades do paciente, planejar e executar as intervenções de enfermagem adequadas. Oferece aos enfermeiros a oportunidade de avaliar e reavaliar suas intervenções.

4 O desenvolvimento de software com as etapas do processo de enfermagem baseado na (Teoria das Necessidades Humana Básica) com linguagens padronizadas que facilitam e fomentam o processo de enfermagem.Tal tecnologia irá contribuir para a incorporação do PE na prática profissional. Como fator limitante, destaca-se que este software foi desenvolvido para pacientes de UTIs adulto, no entanto há possibilidade de criação de outros softwares para outras áreas da Enfermagem.

5 A SAE garante atenção adequada e proporciona assistência integral permitindo melhor qualidade dos cuidados prestados e resolubilidade dos problemas. Fortalece a qualidade do serviço prestado e estimula uma relação terapêutica.

6 A aplicação da SAE contribui com a prática dos enfermeiros de forma singular e diferenciada atuando nos variados níveis de complexidade, buscando promover uma reintegraçãodas pessoas em sofrimento psíquico, além de proporcionar aos clientes o bem estar biopsicossocial. No entanto para que a SAE concretize-se é necessário que haja uma 
transformação desde aspectos institucionais, relações de integração com a equipe multiprofissional e preparo dos profissionais.

7 A aplicação do PE possibilitou o desenvolvimento de uma assistência com embasamento científico, ressalta-se que o uso de uma linguagem uniformizada melhora a prática do cuidado, pois facilita a comunicação. Proporciona maior autonomia do Enfermeiro, organiza o trabalho. As dificuldades identificadas foram: desinteresse em contribuir com a sistemática, deficiência de conhecimento e despreparo dos profissionais para lidar com o método.

8 A utilização do instrumento de implementação da SAE, serve como guia e permite a documentação das ações de forma clara e objetiva, facilitando a comunicação e continuação do cuidado. A aplicação do PE possibilita um cuidado singular e com qualidade.

9 Constatou-se, que o processo sistematizado do cuidado favorece o vínculo entre usuários e enfermeiro, valorizando sua prática profissional e sua atuação perante a equipe multiprofissional. Além da observância de que o método do PE direciona o cuidado para as necessidades específicas de cada usuário, possibilitando uma assistência qualificada e humanizada.

10 Elaboração de instrumento para sistematizar a assistência de enfermagem numa unidade psiquiátrica de um hospital geral, foi proposto um instrumento em forma de check-list, visando possibilitar articulação entre o cuidado de enfermagem baseado na psiquiatria clínica, a abordagem integral das necessidades do paciente e dando condição à enfermeira de tomar decisões.

11 Através da aplicação do processo de enfermagem nos idosos com Alzheimer obteve-se um maior conhecimento do estado de saúde deles. Onde por meio das etapas do PE, foi possível auxiliar no tratamento e prevenir complicações, além de oferecer orientações de enfermagem e transdisciplinar.

12 A sistematização resulta na consolidação da profissão e visibilidade para as ações desempenhadas pelo enfermeiro, bem como oferece o subsídio para o desenvolvimento do conhecimento técnico-cientifico. Minimiza gastos com erros e desperdícios de tempo, otimiza a comunicação entre profissionais. Porém, apesar dos benefícios, observa-se que alguns enfermeiros não a utilizam, visto que tomam decisões não pautadas no raciocínio clínico, assim como não se preocupam com a qualidade dos registros. 
13 Objetivou-se aplicar a sistematização da assistência de enfermagem de forma integralizada e humanizada.O processo de enfermagem possibilitou uma maior interação com a paciente e o conhecimento das implicações do acidente aracnídeo, o que facilitou o planejamento da assistência de forma integralizada. Foi um importante instrumento da prática assistencial uma vez que direcionou o cuidado individualizado atendendo as necessidades da paciente, assim como, uma avaliação posterior da evolução do caso.

14 O alvo principal do trabalho foi levantar as dificuldades encontradas pelo enfermeiro na realização da Sistematização da Assistência de Enfermagem (SAE) na unidade de pronto atendimento. Aimplementação da SAE em unidade de emergência constitui-se de vários desafios desde a alta demanda de clientes, falta de tempo e o desconhecimento.

15 Analisar a sistematização da assistência de enfermagem aplicada a um adolescente hospitalizado por paracoccidioidomicose infanto-juvenil.A sistematização aplicada ao adolescente em questão foi segura, de qualidade e contribuiu, sobremaneira, para sua alta hospitalar, com remissão dos sintomas que o levaram à internação e melhor prognóstico.

16 Construção e validação um instrumento para a Sistematização da Assistência de Enfermagem para adolescentes hospitalizados.Após a construção e validação do referido instrumento, foi elaborada a versão final do instrumento, considerando sua relevância para a prática assistencial. Esse instrumento facilitará a comunicação e o registro da assistência de enfermagem, tornando-a mais efetiva e visível para a clientela e para os outros profissionais da equipe de saúde do hospital.

17 Propor reflexão quanto à importância da aplicação do PE como um instrumento metodológico e sistematizado para a melhoria da qualidade da assistência de enfermagem psiquiátrica, nos diferentes cenários da sua prática profissional.A partir da aplicação do $\mathrm{PE}$, os resultados planejados para a paciente foram alcançados, proporcionando-lhe melhor qualidade de vida, no período em que permaneceu internada.

Fonte: Elaborado pelos próprios autores.

Quadro 3 - Frequência dos eixos encontrados, após leitura dos artigos. Rio de Janeiro, RJ, Brasil, 2020.

Eixos temáticos

\begin{tabular}{lcc}
\hline Aplicabilidade da SAE em diferentes níveis de atenção & 2 Artigos & $11,76 \%$ \\
\hline Aplicabilidade da SAE em diferentes patologias & 9 Artigos & $52,94 \%$ \\
\hline
\end{tabular}


Desenvolvimento tecnologias e instrumentos para a SAE

5 Artigos

$29,41 \%$

Relação da aplicação da SAE e recursos humanos

1 Artigos

$5,89 \%$

Fonte: Elaborado pelos próprios autores.

\section{DISCUSSÃO}

Após a leitura dos artigos e a categorização em eixos temáticos, foi possível realizar duas categorias temáticas abrangentes que compõem a síntese e agrupamento das ideias dos artigos. Sendo estas: Avanços e benefícios da aplicação da Sistematização da Assistência de Enfermagem; Limites e fragilidades na Aplicação da Sistematização da Assistência de Enfermagem.

\section{Avanços e Benefícios da Aplicação}

\section{da Sistematização da Assistência de}

\section{Enfermagem}

Para os autores o a aplicação da Sistematização da Assistência de Enfermagem traz muitos benefícios para a profissão. Os artigos 3,5,8,9,11,13,15,17 trouxeram em comum, a melhora da assistência prestada aos pacientes, uma vez que o cuidado foi individualizado e direcionado ás necessidades de cada individuo e maior resolutividade dos problemas, atrelado a isto ocorre a melhora no prognóstico do pacientes. Tal fato está de acordo com um estudo realizado acerca da percepção dos enfermeiros na aplicação do processo de enfermagem que traz que o PE proporciona maior qualidade à assistência, além de mais eficiência e autonomia na assistência prestada ${ }^{(24)}$.

O fortalecimento da enfermagem quanto profissão e quanto ciência também foi identificado pelos artigos 6,7,9 e 12, que destacaram a importância da implementação da SAE, pois esta traz autonomia profissional e embasamento cientifico para as ações de enfermagem. Esta ideia vai de encontro com as produções disponíveis na literatura, que afirmam que a SAE contribui diretamente para valorização e autonomia do enfermeiro, além de facilitar o desenvolvimento de ações em saúde ${ }^{(25)}$.

Foi possível identificar nos artigos 4,10,16 a formulação de tecnologias para facilitar a aplicação do processo de enfermagem, isto mostra que enfermeiros estão interessados e engajados em não somente aplicar o PE, mas também fomentar subsídios para a sua aplicação. Outro beneficio evidenciado nos artigos 7,8,6 foi a melhora na comunicação entre a equipe multiprofissional e a melhora nos registros dos pacientes que facilitaram a continuidade da assistência.

Outra vantagem identificada foi a redução dos gastos e dos custos da internação do paciente, pois com uma assistência 
sistematizada, o individuo tem os cuidados centrados em suas prioridades e com mais qualidade, melhorando desta forma seu prognóstico e os custos de seu atendimento.

\section{Limites e fragilidades na Aplicação} da Sistematização da Assistência de Enfermagem

A SAE foi instituída em forma de decreto há uma década e no decorrer deste tempo algumas dificuldades foram identificadas, os artigos 1,12 denotam que os registros realizados pelos enfermeiros estavam fragmentados e incompletos, tal fato mostra preocupação com a qualidade destes registros. Uma vez que a evolução e anotação de enfermagem são essenciais para a continuidade do cuidado e para respaldo profissional. A fragilidade dos registros é um problema comum identificado nas produções científicas, que revela a necessidade de revisão da forma que os registros são realizados ${ }^{(26)}$.

A não adesão ou a não realização adequada desta metodologia de cuidado foi observada através dos artigos 7,14, que pontuaram como motivo para estas condutas á falta de interesse profissional em de fato querer fazer e aprender a SAE. E a outra razão esta relacionada com a necessidade de conhecimento da SAE e do PE, que pode ser solucionado com educação permanente e atualizações. Segundo o estudo realizado com enfermeiros, a não adesão pode estar relacionado com o afastamento entre o ensino teórico e a prática profissional. E o conhecimento é essencial para sua execução, pois é através deste que o profissional adquiri autonomia $^{(27)}$.

Os artigos 1,2 apontaram como fragilidades para SAE o baixo quantitativo de recursos humanos que sobrecarrega os enfermeiros, além da alta demanda de pacientes, pois o tempo despendido na execução da SAE implica na necessidade de um dimensionamento adequado de profissionais de enfermagem. De acordo com um estudo realizado com enfermeiros a respeito da $\mathrm{SAE}$, o número reduzido de enfermeiras repercute diretamente nos registros qualidade da assistência prestada ${ }^{(28)}$.

\section{CONCLUSÃO}

Através dos resultados encontrados neste estudo, podemos observar que a SAE se configura em uma grande conquista para enfermagem, pois após uma década de sua regulamentação no Brasil, inúmeros avanços para categoria foram alcançados, seja enquanto enquanto profissão, ciência a até mesmo para os nossos clientes. No entanto ainda há barreiras a serem ultrapassadas, pois 
ainda temos dificuldade em sua

resultados

implementação, observamos que o dimensionamento profissional, treinamento e capacitação interferem diretamente na qualidade dos registros e na assistência realizada. Desta forma para a SAE permaneça com qualidade é necessário envolvimento das instituições de trabalho, do conselho e dos próprios profissionais.

É evidente que este estudo foi muito importante para o nosso conhecimento, compreensão e aprofundamento sobre as nuances desse tema, pois, a SAE faz parte do cotidiano dos enfermeiros e nos desafia diariamente a enfrentarmos as dificuldades com soluções não utópicas. É preciso valorizar os avanços já alcançados ao longo desta última década e ??o conhecimento adquirido sobre a SAE para basear nossas ações como enfermeiros. Também compreendemos melhor os avanços e as limitações de sua implementação na assistência de enfermagem. Permitiu-nos também desenvolver e aperfeiçoar competências de investigação, seleção, organização e comunicação das informações levantadas no presente trabalho. Esperamos ter cooperado de forma positiva para o fomento, suscitação de novos questionamentos e pesquisas sobre a temática proposta. encontrados em nossa pesquisa, destacamos a importância desta temática para a profissão e deste conhecimento para instrumentalizar o profissional para o desenvolvimento de uma assistência qualificada, recomendamos que a SAE seja objeto de discussões em congressos e eventos científicos com o propósito de promover ajustes no que tange as suas limitações no processo do trabalho. Acreditamos que este estudo possa subsidiar novos estudos a partir do perfil traçado sobre os avanços e limites da SAE na última década (2009-2019).

\section{REFERÊNCIAS}

1. Leite de Barros ALB, Lopes JL. A legislação e a sistematização da assistência de enfermagem. RevEnferm em Foco [Internet]. 2010 [acesso em 05jan 2020];1(2):63-5. Disponível em:http://revista.cofen.gov.br/index.php/e nfermagem/article/viewFile/17/18

2. Silva DG, Freiberger MF, Silva JL, Vale JS, Gonçalves JCR. O Marco de Wanda Aguiar Horta para o processo de Enfermagem no Brasil. Rev Cie Fac Edu Mei [Internet]. 2011 [Acesso em 05 jan 2020]; 2(Supl-I):56-9. Disponível em: http://www.faema.edu.br/revistas/index.p hp/Revista-FAEMA/article/view/68/299

3. Conselho Regional de Enfermagem de São Paulo. Processo de Enfermagem: Guia para a prática. $1^{a}$ ed. São Paulo, SP; 
2015. Disponível em: https://portal.corensp.gov.br/sites/default/files/SAE-web.pdf

4. Conselho Federal de Enfermagem. Resolução COFEN-358/2009.

Sistematização da Assistência de Enfermagem e a implementação do Processo de Enfermagem em ambientes, públicos ou privados, em que ocorre o cuidado profissional de Enfermagem. Brasília, DF; 15 out 2009.

5. Mendes KDS, Silveira RCCP, Galvão CM. Revisão Integrativa: método de pesquisa para a incorporação de evidências na saúde e na enfermagem. Rev Texto e Contexto-enferm. 2008 [acesso em 12 jan 2020]; 17(4): 758-64. Disponível em:http://www.scielo.br/scielo.php?script $=$ sci_arttext $\&$ pid $=$ S010407072008000400018

6. Bardin L. Análise de conteúdo. Edições 70. São Paulo; 2011. p. 74-105.

7. Neves RS, Shimizu HE. Análise da implementação da Sistematização da Assistência de Enfermagem em uma unidade de reabilitação. Rev. bras. Enferm [internet]. 2010 [acesso em 16 jan 2020]; 63(2):222-9.Disponível em: http://www.scielo.br/pdf/reben/v63n2/09. pdf

8. Almeida MA, Severo IM, Chaves EB, Barreto LNM, Borba DM. Tempo despendido na execução do processo de enfermagem em um centro de tratamento itensivo. Esc. Anna Nery. 2012 [acesso em 16 jan 2020]; 16(2):292-6. Disponível em:

http://www.scielo.br/pdf/ean/v16n2/12.pd $\mathrm{f}$

9. Corso NAA, Gondin APS, D'Almeida PCD, Albuquerque MGF. Sistematização da Assistência de Enfermagem para acompanhamento ambulatorial de pacientes com esclerose múltipla. Rev. Esc. Enferm. USP. 2013 [acesso em 16 jan 2020]; 47(3)750-5. Disponível em: http://www.scielo.br/pdf/reeusp/v47n3/00 80-6234-reeusp-47-3-00750.pdf

10. Martins MCT, Chianca TCM. Construção de um software com o Processo de Enfermagem em Terapia Intensiva. J. Health Inform. 2016 [acesso em 16 jan 2020]; 8(4)119-25. Disponível em: http://www.jhi-sbis.saude.ws/ojsjhi/index.php/jhi-sbis/article/view/420/274

11. Rocha RC, Bezerra MAR, Rocha JC, Rocha MC, Gonçalves CB, Cardoso ARS. Processo de enfermagem aplicado a paciente submetida à histerectomia: relato de experiência. Rev. Enferm. da UFPI. 2015[acesso em 16 jan 2020]; 4(3):86-90. Disponível em: https://revistas.ufpi.br/index.php/reufpi/art icle/view/1879/pdf

12. Monteiro ARM, Martins MGQ, Lobô AS, Freitas CA, Barros KM, Vale SF. Sistematização da assistência de enfermagem à criança e ao adolescente em sofrimento psíquico. $\mathrm{J}$ res.: fundam care. 2015[acesso em 16 jan 2020]; 7(4):318596. Disponível em: http://www.seer.unirio.br/index.php/cuida dofundamental/article/view/3500/pdf_169 2

13. Silva RS, Pereira A, Conceição JC, Biai ISC. Aplicação do Processo de Enfermagem: Estudo de caso com um portador do vírus da hepatite C. Rev. Baiana de Enferm. 2010[acesso em 16 jan 2020]; 24(1,2,3):87-95. Disponível em: https://portalseer.ufba.br/index.php/enfer magem/article/view/5547/4027

14. Gomes IP, Nóbrega MML, Collet N, Fernandes MGM, Araújo YB, Lima KA. Processo de Enfermagem ao adolescente 
portador de anemia falciforme. Rev. Brasileira de cienc. da saúde. 2011[acesso em 16 jan 2020]; 15(4):461-64. Disponível em: https://periodicos.ufpb.br/ojs/index.php/rb cs/article/view/10038/6861

15. Schmalfuss JM, Scholz DCS, Prates LA. A aplicação do processo de enfermagem no atendimento a uma mulher com artrite reumatoide. Rev. Enferm. UFPE. 2013[acesso em 16 2020]; 7(esp):1023-9. Disponível em: https://periodicos.ufpe.br/revistas/revistae nfermagem/article/view/11571/13561

16. Toledo VP, Motobu SN, Garcia APRF. Sistematização da assistência de enfermagem em unidade de internação psiquiátrica. Rev. Baiana de Enferm. 2015[acesso em 16 jan 2020]; 29(2):1729. Disponível em: https://portalseer.ufba.br/index.php/enfer magem/article/view/11707/pdf_130

17. Mattos CMZ, Garces SBB, Costa FTL, Rosa CB, Brunelli AV, Hansen D et al. Processo de enfermagem aplicado a idosos com Alzheimer que participam do projeto estratégias de reabilitação. Estd. Interdiscipl. Envelhc. 2011[acesso em 16 jan 2020]; 16(esp):433-47. Disponível em: https://seer.ufrgs.br/RevEnvelhecer/article /view/17921/16307

18. Truppel TC, Meier MJ, Calixto RC, Peruzzo AS, Crozeta K. Sistematização da Assistência de Enfermagem em unidade de terapia intensiva. Rev. Bras. Enferm. 2009[acesso em 16 jan 2020]; 62(2):221$7 . \quad$ Disponível em: http://www.scielo.br/pdf/reben/v62n2/a08 v62n2.pdf

19. Kamimura HM, Paiva BSR, Ayres JÁ. Sistematização da Assistência de Enfermagem: acidente por Loxosceles gaúcho. Rev. Bras. Enferm. 2009[acesso em 16 jan 2020]; 62(6):928-31.

Disponível

em:

http://www.scielo.br/pdf/reben/v62n6/a22 v62n6.pdf.

20. Félix NN, Rodrigues CDS, Oliveira VDV. Desafios encontrados na realização da sistematização da assistência de enfermagem (SAE) em unidade de pronto atendimento. Arq. Ciênc. Saúde. 2009[acesso em 16 jan 2020]; 16(4):15560. Disponível em: http://repositorioracs.famerp.br/racs_ol/vol-164/IDK2_out-dez_2010.pdf

21. Junior EFP, Pire AS, Jomar RT, Peres EM, Gomes AMT, Rocha AC. Sistematização da assistência de enfermagem aplicada a um adolescente hospitalizado por paracoccidioidomicose. Rev. Enferm. UERJ. 2015[acesso em 16 jan 2020]; 23(6): 767-72. Disponível em: https://www.e-

publicacoes.uerj.br/index.php/enfermage muerj/article/view/18764/16177

22. Marques DKA, Nóbrega MML. Instrumento de Sistematização da Assistência de Enfermagem para Adolescentes Hospitalizados. Rev. Min. Enferm. 2009[ acesso em 16 jan 2020]; 13(3):372-80. Disponível em: http://www.reme.org.br/artigo/detalhes/20 2.

23. Toledo VP, Ramos NA, Wopereis F. Processo de Enfermagem para pacientes com Anorexia Nervosa. Rev. Bras. Enferm. 2011[acesso em 16 jan 2020]; 64(1):193-7. Disponível em: http://www.scielo.br/pdf/reben/v64n1/v64 n1a29.pdf

24. Benedt AS, Gelbcke FL, Amante LN, Padilha MIS, Pires DP. Processo de enfermagem: instrumento da sistematização de enfermagem na percepção dos enfermeiros. J. 
res.:fundam. Care. 2016[acesso em05 mar 2020]; 8(3):4780-8. Disponível em: http://www.seer.unirio.br/index.php/cuida dofundamental/article/view/4237/pdf_1

25. Cavalcante JRC, Amaral MS, Loreto RGO. Sistematização da assistência em enfermagem: perspectivas para 0 fortalecimento da enfermagem do trabalho. Revista Científica FacMais. 2017[acesso em 08 mar 2020]; 9(2):147$60 . \quad$ Disponível em: http://revistacientifica.facmais.com.br/wpcontent/uploads/2017/09/7.-

SISTEMATIZA\%C3\%87\%C3\%83O-DAASSIST\%C3\%8ANCIA-EMENFERMAGEM-PERSPECTIVASPARA-O-FORTALECIMENTO-DAENFERMAGEM-DO-TRABALHO.pdf

26. Maziero VG, Vannuchi MTO, Haddad MCL, Vituri DW, Tada CN. Qualidade dos registros dos controles de enefermagem em um hospital universitário. Rev.Min. Enferm. 2013[acesso em 09 mar 2020]; 17(1):166$77 . \quad$ Disponível em: http://www.reme.org.br/artigo/detalhes/58 7

27. Silva EGC, Oliveira VC, Neves GBC, Guimarães TMR. O conhecimento do enfermeiro sobre a Sistematização da Assistência de Enfermagem: da teoria à prática. Rev. esc. Enferm. USP. 2011[acesso em 09 mar 2020]; 45(6):1380-6. Disponível em: http://www.scielo.br/pdf/reeusp/v45n6/v4 5n6a15.pdf

28. Pimpão FD, Filho WDL, Vaghetti HH, Lunardi VL. Percepção da Equipe de Enfermagem sobre seus registros: buscando a sistematização da assistência de enfermagem. Rev. Enferm. UERJ. 2010[acesso em: 10 mar 2020]; 18(3):405-10. Disponível em: http://www.facenf.uerj.br/v18n3/v18n3a1 2.pdf

Submissão: 2021-01-07

Aprovado: 2021-03-31 